\title{
Article
}

Subscriber access provided by University Library of Southern Denmark

\section{Measurement of free drug concentration from biological tissue by Solid-phase Microextraction: In-Silico and Experimental Study}

Mohammad Huq, Marcos Tascon, Emir Nazdrajic, Anna Roszkowska, and Janusz Pawliszyn

Anal. Chem., Just Accepted Manuscript • DOI: 10.1021/acs.analchem.9b00983 • Publication Date (Web): 15 May 2019

Downloaded from http://pubs.acs.org on May 15, 2019

\section{Just Accepted}

"Just Accepted" manuscripts have been peer-reviewed and accepted for publication. They are posted online prior to technical editing, formatting for publication and author proofing. The American Chemical Society provides "Just Accepted" as a service to the research community to expedite the dissemination of scientific material as soon as possible after acceptance. "Just Accepted" manuscripts appear in full in PDF format accompanied by an HTML abstract. "Just Accepted" manuscripts have been fully peer reviewed, but should not be considered the official version of record. They are citable by the Digital Object Identifier (DOI®). "Just Accepted" is an optional service offered to authors. Therefore, the "Just Accepted" Web site may not include all articles that will be published in the journal. After a manuscript is technically edited and formatted, it will be removed from the "Just Accepted" Web site and published as an ASAP article. Note that technical editing may introduce minor changes to the manuscript text and/or graphics which could affect content, and all legal disclaimers and ethical guidelines that apply to the journal pertain. ACS cannot be held responsible for errors or consequences arising from the use of information contained in these "Just Accepted" manuscripts. 


\title{
Measurement of free drug concentration from biological tissue by Solid- phase Microextraction: In-Silico and Experimental Study
}

\author{
Mohammad Huq, Marcos Tascon ${ }^{\ddagger}$ Emir Nazdrajic, Anna Roszkowska ${ }^{\dagger}$ Janusz Pawliszyn* \\ Department of Chemistry, University of Waterloo, Waterloo, Ontario N2L 3G1, Canada \\ *Email janusz@uwaterloo.ca, Fax: 519-746-0435; Tel: 519-888-4567
}

\begin{abstract}
In this article, the use of an SPME technique is reported for the first time for direct measurement of free drug concentration in solid tissue. In our investigations, we considered doxorubicin (DOX) spiked in homogenized tissue matrix at transient and equilibrium extraction conditions, with subsequent assessment of obtained experimental results by an in-silico approach using mathematical models developed in COMSOL Multyphysics. In-silico studies were performed based on transported diluted species (tds) and reaction engineering (re) modules from COMSOL Multiphysics, using the same conditions as those used to attain experimental results. To determine the apparent binding affinity of DOX to the tissue matrix which contains multiple binding species, the experimentally determined binding affinity of DOX with human serum albumin (HSA) was considered to simplify the mathematical calculations. Here, the value of the binding affinity was considered for single binding site and adjusted by fitting the experimental results with the mathematical model. Bovine lung tissue homogenate was selected as a surrogate matrix, and a biocompatible C-8 commercial SPME fiber was used for extraction of DOX. In total, four mathematical models were herein developed to describe the mass transfer kinetics of solid coatings: in agar gel at static conditions, in PBS solution with agitated conditions, extraction in PBS solution in the presence of an HSA binding matrix, and static extraction in homogenized lung tissue. For all conditions, simulated results were in good agreement with experimental results. The developed mathematical model allows for measurements of free drug concentrations inside tissue matrix, and facilitates calculations of local depletion of DOX by a solid SPME coating. Results of the investigations indicate that local depletion of the free form of DOX, even at the kinetic stage, is negligible for tissue extraction, as the release of the heavily bound analyte (over $99 \%$ binding to tissue matrix) is very rapid, thus easily compensating for the loss of the drug to the SPME coating. This indicates that the dissociation rate constant of DOX from lung tissue components is very rapid; therefore, the mass transfer of drug to the fiber coating via free from is very efficient. Our results also indicate that thin coating SPME fibers provide a good way to measure drug distribution after dosing, as extractions via thin coating SPME fibers do not affect the free concentration of the drug, which is responsible for drug distribution in tissue.
\end{abstract}

One of the main aims of clinical pharmacology studies is to enable the most accurate measurements of drug concentrations in living systems, a task most likely to succeed via in vivo assessments of free drug concentrations at receptor sites (the biophase). Given that the majority of drug-receptor interactions take place in tissue, determining free drug concentrations at the intracellular space would be a more rational way to investigate the pharmacokinetics/pharmacodynamics (PK/PD) of a given drug, as compared to its measurement in blood plasma. ${ }^{1,2}$ These concerns are of particular importance in PK/PD studies involving drugs that are administered subcutaneously (SC) or intramuscularly (IM), as the active concentration of such drugs would be significantly higher in tissue in comparison to that present in intravenous (IV) or oral administration.. In such cases, the in vivo free concentration of the administered drug at the intracellular space would be the most appropriate indicator of therapeutic efficiency. ${ }^{3}$ However, given the complex nature of drug distribution in tissue matrix as well as the limitations of traditional analytical techniques available for such measurements, in vitro measurement of free drug concentrations in blood plasma is oftentimes adopted as a surrogate technique to study PK/PD. ${ }^{4-6}$ While a variety of analytical methods are available for measurement of free drug concentrations in tissue matrix, such as equilibrium dialysis, ultrafiltration, and microdialysis; ${ }^{7-10}$ few of them are capable of in vivo measurement. Microdialysis (MD) is the method most widely used to monitor free analyte concentrations in the extracellular space of tissue. However, the MD technique is burdened by major drawbacks, such as very low recovery for highly bound nonpolar drugs, and low temporal resolution of fast changing tissue components. The electrochemical biosensors technique, on the other hand, is highly efficient for measurement of rapid changing tissue components, since it promotes fast detection via chemical or biochemical reactions. However, this technique, already limited to the detection of electroactive analytes only, is also characterized by low specificity due to the presence of non-electroactive interferences. ${ }^{9,11}$ Due to technical difficulties, equilibrium dialysis and ultrafiltration are only used for in vitro analysis in blood plasma and other biological fluids. ${ }^{12,13}$ In this manner, equilibrium dialysis encounters its major experimental limitations in the longer equilibration times (typically $4-28 \mathrm{~h}$ ), volume shifts and poor drug solubility. On the other side, the ultrafiltration technique enables nonspecific interactions with matrix components and equilibrium displacements, producing in this way a bias stemming from overestimations of the free concentration value. ${ }^{14}$ Given the variety of shortcomings associated with MD and electrochemical biosensors, a simpler and more robust analytical technique is thus needed to tackle measurements of drug concentrations in tissue. Solid-phase microextraction (SPME), developed in $1990,{ }^{15}$ stands as particularly suitable for this application given is unique characteristics. In vivo SPME for analysis of tissue can be performed by placing a biocompatible microextraction phase coated onto a thin fiber into tissue matrix with minimal invasion. Here, unbound analytes are selectively extracted from the matrix by diffusion through the boundary layer. Due to the negligible depletive nature of the microextraction phase, SPME does not disturb the binding equilibrium between the analyte and the matrix. Further, SPME allows for extractions to be carried out either in the equilibrium or pre-equilibrium regime. ${ }^{16,17}$ These qualities of SPME render the technique as an ideal choice for measurements of free concentration of analytes from complex biological matrices, as supported by its many 
successful applications in the biological field, both in vivo and ex vivo. ${ }^{18-20}$ In 2003 , Lord et al. first reported the use of fiber SPME for in vivo monitoring of benzodiazepines in the systemic blood circulation of dogs. ${ }^{21}$ Since then, in vivo SPME applications have been widely exploited to study drug biomarkers and metabolomes due to the advantages associated with its low-invasive direct extraction capabilities, as well as its ability to extract analytes with wide range of polarity, an advantage of SPME often referred to as balanced coverage. ${ }^{22-25}$ From a theoretical point of view, Musteata et al. explained the mechanism of SPME extraction of free analytes in the presence of a binding matrix under equilibrium conditions, where the unbound portion of the analyte partitions to the liquid extraction phase of the SPME fiber. ${ }^{26}$ In addition to the use of experimental techniques, mathematical modelling has been demonstrated as an excellent tool to determine the kinetics of SPME extraction in the presence of a binding matrix, as it allows for better predictions of results while minimizing the number of experiments that must be carried out during method development and routine analysis. ${ }^{17,27,28}$ Alam et al. first demonstrated the effects of a binding matrix on the extraction kinetics of SPME liquid coatings via development of a $2 \mathrm{D}$ model in COMSOL Multiphysics. ${ }^{17}$ Gorecki et al. and Zhou et al., in turn, demonstrated the extraction kinetics of solid coatings with the use of Langmuir's isotherms, which assumes isotropy of the fiber surface. ${ }^{29,30}$ Since most of the biocompatible microextraction phases currently employed in SPME experiments for biological applications are consisted of a solid coating, the current work has focused on developing mathematical simulations of SPME kinetics in tissue matrix for solid coatings. To the best of our knowledge, this is the first time a mathematical model to measure free drug concentrations from tissue matrix via SPME is reported in the literature. Of note, the developed model, which allows for calculations of binding constants and free concentrations of relevant drugs directly from tissue, opens an exciting new area of study in bioanalytical chemistry.

Doxorubicin Case Study Doxorubicin (DOX) is a widely known chemotherapeutic agent that has been used for over 40 years used in the treatment of many different types of cancers. The mode of action reveals that DOX causes potential cytotoxic effects by intercalating with DNA base pairs, which limits the proliferation of malignant tissue. However, this drug is highly nonselective in nature, requiring local administration to the site of the malignant tissue as a way to mitigate adverse side effects. Since the site of action of DOX is intracellular and it enters into the cell through passive diffusion like other small molecules, the drug has been commonly administered intravenously (IV). ${ }^{31,32}$ Pharmacokinetic studies have shown that DOX has a plasma half-life of 3-5 min, and a tissue half-life of 24-36 h. This indicates rapid uptake of DOX by tissue matrix. However, passive diffusion of DOX depends on the free form of DOX in the extracellular matrix, where it is heavily bound with multiple extracellular species. To the best of our knowledge, no studies have been reported estimating the free concentration of DOX in tissue matrix. ${ }^{33}$ More recently, DOX has been under preclinical study for treatment of metastatic lung cancer through in vivo lung perfusion (ILVP), where DOX concentration in lung tissue was determined by measurement of total drug concentrations in perfusate solution and serum. ${ }^{4}$ In these scenarios, it is of great bioanalytical interest to develop a novel technique capable of determining the free concentration of a drug in biological tissue by direct sampling, with minimum invasiveness so as to also enable in vivo applications. The current work presents the SPME extraction kinetics for solid coatings in tissue using numerical and experimental models. As a proof of concept, DOX was selected as a model drug, while bovine lung tissue was selected as biological matrix. The physics of the biological matrix were also modeled based on fundamental and biological parameters such as tortuosity and intracellular space of tissue matrix, since these factors control drug diffusion and distribution. Experimental results were then attained and compared with the developed numerical simulations. Once optimized, the model enabled calculations of free drug concentrations as well as relevant concepts for in vivo sampling, such as sampled area, depleted area, and spatial resolution.

Theoretical Considerations SPME extraction takes place via free concentration. If the amount extracted is negligible, then the equilibrium concentration of the analyte in the sample matrix is assumed to be equivalent to the free concentration, which is measured according to the following equation:

$[A]^{\text {free }}=\frac{\mathrm{n}}{f}$

Where $\mathrm{n}$ is the amount of analyte extracted on the fiber coating at equilibrium and $\mathrm{f}$ is the fiber constant. The value of $f$ is then given by eq. (2):

$f=K_{e s} * V_{f}$

Where, $V_{f}$ is the volume of the extraction phase (in $\mathrm{m}^{3}$ ) and $K_{e s}$ is the partition coefficient of the analyte (dimensionless). $K_{e s}$ is derived from the equilibrium extraction equation Eq. (3):

$K_{\text {es }}=\frac{[A]_{\text {fiber }}^{e q}}{[A]_{\text {sample }}^{e q}}$

However, when solid coatings are employed in SPME, analytes are adsorbed on the active site of the surface area rather than partitioned, making the above eq. (2) inadequate for measurements of free concentrations under these conditions. A theoretical approach based on Langmuir's theory for the extraction kinetics of solid coating SPME has been previously addressed in the literature..$^{28,34} \mathrm{In}$ brief, the amount of extract on the solid coating at equilibrium $c s^{e q}\left(\mathrm{~mol} . \mathrm{m}^{-2}\right)$ is given by:

$C S^{e q}=\frac{\gamma_{\mathrm{s}} * K *[A]^{e q}}{1+K *[\mathrm{~A}]^{e q}}$

Where $\gamma_{s}$ is the maximum free active-site concentration in the fiber $\left(\mathrm{mol} . \mathrm{m}^{-2}\right), K$ is the adsorption equilibrium concentration $\left(\mathrm{m}^{3} \cdot \mathrm{mol}^{-1}\right)$, and $[\mathrm{A}]^{\mathrm{eq}}$ is the free concentration of analyte in the matrix at equilibrium $\left(\mathrm{mol} . \mathrm{m}^{-3}\right)$.

The mass transfer in solid coatings is defined by Langmuir's isotherm, which is defined as:

$r_{a d s}-r_{d e s}=k_{a d s} *[A] *(1-\theta)-k_{d e s} * \theta$

Where, $r_{a d s}$ is the rate of adsorption of analyte onto the coating surface $\left(\mathrm{mol} . \mathrm{m}^{-2} \cdot \mathrm{s}^{-1}\right) ; r_{\text {des }}$ is the rate of desorption $\left(\mathrm{mol} . \mathrm{m}^{-2} \cdot \mathrm{s}^{-1}\right)$;

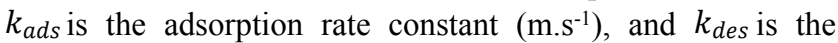
desorption rate constant $\left(\mathrm{mol} \cdot \mathrm{m}^{-2} \cdot \mathrm{s}^{-1}\right)$. For solid coatings, the rates of adsorption and desorption are dependent on the fraction of vacant sites available on the solid surface, which is defined 
as $\theta$. The fraction of occupied sites on the solid surface is defined as:

$\theta=\frac{c s}{\gamma_{s}}$

Where $c s$ is the amount extracted on the fiber at a given time t, and $\gamma_{\mathrm{s}}\left(\mathrm{mol} . \mathrm{m}^{-2}\right)$ is the maximum active surface concentration. The latter can be defined as follows:

$\gamma_{\mathrm{S}}\left(\frac{m o l}{m^{2}}\right)=\frac{\text { saturated amount extracted on the fiber }}{\text { active surface area per fiber }}=\frac{c s_{\text {sat }}}{\mathrm{A}}$

Eq. (5) can be rearranged as:

$r_{a d s}-r_{d e s}=k_{a d s} *[A] *\left(1-\frac{c s}{\gamma_{s}}\right)-k_{d e s} * \frac{c s}{\gamma_{s}}$

At equilibrium, the rates of adsorption and desorption are equal. Therefore, eq. (5) can be expressed as:

$K=\frac{k_{a d s}}{k_{d e s}}=\frac{c s^{e q} / \gamma_{\mathrm{s}}}{[\mathrm{A}]^{e q} *\left(1-\mathrm{cs} / \gamma_{\mathrm{s}}\right)}$

Eq. (7) provides the partition coefficient value for an analyte in a given solid coating. However, if the extraction amount is significantly high, significant depletion of the free concentration of the analyte in the local area of the fiber will occur after the system reaches equilibrium. If we consider the extraction of analyte without any binding matrix present in the system, the free concentration will be same as the total concentration. Therefore, eq. (1) can be expressed as follows:

$[A]^{\text {free }}=[A]^{e q}+\frac{\mathrm{n}}{V_{s}}$; where $\frac{\mathrm{n}}{V_{s}}$ is depletion concentration (8)

And equations (7) and (8) can be rewritten as:

$[A]^{\text {free }}=[A]^{e q}+\frac{n}{V_{s}}=\frac{c s^{e q} / \gamma_{s}}{K *\left(1-c s / \gamma_{s}\right)}+\frac{n}{V_{s}}$

$[A]^{\text {free }}=\frac{c s^{e q}}{K *\left(\gamma_{\mathrm{s}}-c s\right)}+\frac{n}{V_{s}}$

Eq. (10) provides the free concentration of analyte extracted by a solid coating without the presence of a binding matrix.

In the presence of a single binding matrix and univalent binding site, analyte $\mathrm{A}$ is in equilibrium with the matrix component $\mathrm{M}$, where the binding association constant $K_{A}$ is given by:

$A+M=A M$

$K_{A}=\frac{k_{f}}{k_{r}}=\frac{[A M]}{[A]^{e q} *[M]}$

Where, $k_{f}, k_{r}$, and $[A]^{e q}$ are the rate of association, rate of dissociation, and concentration of $A$ at equilibrium, respectively. $[M]$ is the concentration of the binding matrix, and $[A M]$ is the concentration of analyte bound to the matrix. Eq. (11) can be expressed as:

$K_{A}=\frac{[\mathrm{AM}]}{[\mathrm{A}] *[\mathrm{M}]}=\frac{[A]^{\text {tot }}-[A]^{\text {free }}}{[A]^{\text {free }} *\left([\mathrm{M}]^{\text {tot }}-[A]^{\text {tot }}+[A]^{\text {free }}\right)}$
Where, $[A]^{\text {tot }}$ is the total concentration of analyte or initial concentration, $[A]^{\text {free }}$ is the free concentration of analyte, and $[M]^{\text {tot }}$ is the total matrix concentration.

If the binding matrix concentration $[\mathrm{M}] \gg[\mathrm{A}]^{\text {tot }}$, then eq. (12) can be rearranged as:

$K_{A} *[M]^{\text {tot }}=\frac{[A]^{\text {tot }}}{[A]^{\text {free }}}-1$

A linear regression line can be obtained from eq. (13), where the slope gives the value of the binding association constant $K_{A}$. Equation (8) describes the mass transfer kinetics of free analyte to the extraction phase in absence of any binding matrix. However, in presence of binding matrix the mass transfer kinetics of free analyte will be same since it depends on the partition coefficient $K$. Therefore, we can introduce the $[A]^{\text {free }}$ by combining the eqs. (8) and (13):

$$
\begin{gathered}
K_{A} *[M]^{\text {tot }}=\frac{[A]^{\text {tot }}}{\left([A]^{e q}+\frac{n}{V_{S}}\right)}-1 \\
\frac{1}{[A]^{\text {free }}}=\left(K_{A} *[M]^{\text {tot }}+1\right) * \frac{1}{[A]^{\text {tot }}}
\end{gathered}
$$

The binding association constant can thus be calculated by using eq. (14). Interestingly, the product of $K_{A \cdot} \cdot[M]^{t o t}$ from eq. (14) is a unitless constant, which we can consider as the apparent binding constant $K_{A p p}=K_{A \cdot} \cdot[M]^{t o t}$. In this paper, we adopted eq. (14) for the binding study of DOX with tissue binding matrix. For mathematical simplification, we considered univalent binding site of DOX with a single binding species which is HSA in this case study. However, we justified the mathematical limitations by adjusting the experimental results with simulations. For experimental results we extended the equilibrium conditions described above by the exact equations to transient situations prior to equilibrium using numerical modeling.

\section{EXPERIMENTAL SECTION}

Materials and Supplies. Human Serum Albumin (HSA), doxorubicin hydrochloride (DOX), formic acid (FA), and ammonium acetate (LC-MS grade) were purchased from Sigma-Aldrich (Oakville, ON, Canada). Agar gel, methanol $(\mathrm{MeOH})$, and water were LC-MS grade and purchased from Fisher Scientific (Mississauga, ON, Canada). C-8 mixed mode SPME fibers were kindly provided by Millipore-Sigma (Oakville, ON, Canada). A phosphate-buffered saline solution (PBS) at $\mathrm{pH} 7.4$ was prepared according to the standard procedure described in the Supporting Information (section 1). Standard stock solutions were prepared in methanol at a concentration of $2000 \mu \mathrm{g} \cdot \mathrm{mL}^{-1}$ and stored at $-80{ }^{\circ} \mathrm{C}$. Bovine lung tissue was purchased from a local meat shop, and respective experiments were conducted with the approval of the University of Waterloo's Office of Research Ethical Board.

Measurement of Maximum Surface Concentration of DOX $\left(\gamma_{s}\right)$. According to eq. (9), the maximum site concentration of doxorubicin on the fiber coating is attained once equilibrium is reached. To experimentally attain a measurement of the 
maximum surface concentration of DOX on a C-8 mixed mode SPME fiber, extractions were performed at $0.5,1,2,3,4$, and 5 $\mu \mathrm{g} \cdot \mathrm{mL}^{-1}$ initial concentrations in PBS under agitation at 1500 $\mathrm{rpm}$. The active surface area of the fiber coating was determined via Brunauer-Emmett-Teller (BET) analysis (S2).

Measurement of the Adsorption Equilibrium Constant (K) for DOX The adsorption equilibrium constant $(K)$ for doxorubicin onto a C-8 mixed mode SPME substrate was attained by carrying out extractions under the following conditions: agitated equilibrium extractions from PBS were carried out using two different initial concentrations (Supporting Information; section 4). Doxorubicin was spiked at $100 \mathrm{ng} \cdot \mathrm{mL}^{-1}$ and $50 \mathrm{ng} \cdot \mathrm{mL}^{-1}$ in PBS at $\mathrm{pH}$ 7.4. Extractions were performed at $1500 \mathrm{rpm}$ with preconditioned SPME C-8 mix mode fibers. Fibers were preconditioned with $\mathrm{ACN} / \mathrm{H}_{2} \mathrm{O}$ (80/20) for 30 mins. An extraction time profile was obtained at eight different time points by independent triplicates, within a 120 min period. After each extraction time step, fibers were gently cleaned with Kim wipes and rinsed with $300 \mu \mathrm{L}$ of LCMS grade water for $10 \mathrm{~s}$ to remove salts and unspecific attachments from the coating. Desorption was performed with $300 \mu \mathrm{L}$ of $\mathrm{ACN} / \mathrm{H}_{2} \mathrm{O}(80 / 20+0.1 \% \mathrm{FA})$ for $60 \mathrm{~min}$ in a shaker at $1500 \mathrm{rpm}$.

Static Extraction Time Profile of Doxorubicin in Agarose Gel: In this experiment, we evaluated the kinetic profile for static extraction of DOX in the absence of a binding matrix. Agarose powder $(0.8 \%, \mathrm{w} / \mathrm{w})$ was added in PBS (1X, $\mathrm{pH} 7.4)$ spiked with $100 \mathrm{ppb}$ of DOX. The mixture was heated at $70^{\circ} \mathrm{C}$ in a water bath for $30 \mathrm{~min}$. Once the agarose powder was completely dissolved, the solution was transferred to $2 \mathrm{~mL}$ glass vials and allowed to cool at room temperature until it attained a gel consistency. Then, a preconditioned SPME fiber was inserted into the vial containing $1.8 \mathrm{~mL}$ of agarose gel. Static extraction was performed at eight different time points for 120 hrs. After each extraction time step, fibers were cleaned with Kim wipes and vortexed with $300 \mu \mathrm{L}$ of LC-MS grade water for $10 \mathrm{~s}$ to remove matrix components from the coating. Desorption was performed with $300 \mu \mathrm{L}$ of ACN/H2O (80/20+ $0.1 \% \mathrm{FA}$ ) for $60 \mathrm{~min}$ in a shaker at $1500 \mathrm{rpm}$.

Agitated Extraction Time Profile of Doxorubicin with HSA Binding Matrix. Human Serum Albumin (HSA) was added $(3.6 \%, \mathrm{w} / \mathrm{w})$ in PBS at $\mathrm{pH} 7.4$ spiked with $100 \mathrm{ng} \cdot \mathrm{mL}^{-1}$ of DOX. In order to ensure the analyte sufficiently bound to the matrix, the solution was incubated overnight at room temperature at $250 \mathrm{rpm}$. An extraction time profile was attained by following the same time levels established for PBS samples.

Measurement of Binding Association Constant $K_{A}$ with HSA. Different concentrations of HSA were prepared in PBS and spiked with $100 \mathrm{ng} \cdot \mathrm{mL}^{-1}$ of doxorubicin, then submitted to overnight incubation at $4^{0} \mathrm{C}$ for binding. In order to investigate the effect of the HSA binding matrix and subsequently attain free concentration measurements, equilibrium extractions were performed using preconditioned C-8 mixed mode SPME fibers for five different HSA concentrations.

Extraction of Doxorubicin from Homogenized Lung Tissue. Bovine lung was cut into small pieces and homogenized using dry ice and a meat blender. Doxorubicin was spiked into homogenized lung tissue ( $10 \mathrm{~g}$ of each sample) at concentration levels $5,10,15,25$, and $50 \mu \mathrm{g} \cdot \mathrm{mL}^{-1}$. After spiking, samples were gently vortexed at $500 \mathrm{rpm}$ for 2 hours and then stored at
$4^{0} \mathrm{C}$ overnight for binding. The following day, each sample was equilibrated at room temperature for an hour. Extractions were performed from $1 \mathrm{~g}$ of tissue sample for 25 mins under equilibrium conditions. After extractions, fibers were properly cleaned (wiped with Kimwipes) and rinsed with $300 \mu \mathrm{L}$ of Milli Q water for $10 \mathrm{sec}$ to remove attached tissue matrix and other unspecific matrix components, then submitted to desorption in a vial with $300 \mu \mathrm{L}$ of desorption solvent $\left(80 \% \mathrm{ACN}+20 \% \mathrm{H}_{2} \mathrm{O}\right.$ $+0.14 \%$ FA) for 60 minutes.

LC-MS Characterization of Doxorubicin. DOX (MW: 543.4 Da) was characterized using a Thermo Ultima 3000 liquid chromatographic system coupled to a Quantiva (Thermo Scientific, San Jose, CA) triple quadrupole mass spectrometer. The LC-MS method was carried out according to recently published literature. ${ }^{35}$ Briefly, liquid chromatographic separation was carried out using a C-18 $(4.6 \mathrm{~mm} ; 100 \mathrm{~mm}, 5 \mu \mathrm{m})$ Phenomenex column with mobile phase A $\left(100 \% \mathrm{H}_{2} \mathrm{O}+0.1 \%\right.$ FA) and mobile phase $\mathrm{B}(100 \% \mathrm{ACN}+0.1 \% \mathrm{FA})$, as stated in the associated literature. ${ }^{35}$ The instrumental limit of quantification (LOQ) under these conditions was $0.1 \mathrm{ppb}$.

Mathematical Model for Extraction Time Profile on Solid Coating. In-silico studies were performed using COMSOL Multiphysics 5.3, a finite element method (FEM) based packages software that simulates mass transport using timedependent partial derivatives. For simulation of SPME extraction kinetics, the following modules were employed: transported diluted species (tds), reaction engineering (re) modules under Chemical Species Transport physics, and the laminar flow (lf) module under Fluid Flow physics. Threedimensional models were developed for four different extraction conditions: static extraction in agarose gel, agitated extraction in PBS, agitated extraction in the presence of an HSA binding matrix, and static extraction in lung tissue. The variables and parameters used in these models were obtained from experimental studies, with the exception of the value of the diffusion coefficient, which was adjusted based on reports from the literature ${ }^{36,37,38}$ All the parameters and variables used for simulations are detailed in the Supporting Information (section 2).

\section{RESULTS AND DISCUSSION}

In-Silico Study of Extraction Kinetics under Static Conditions (in Agarose Gel). Agarose gel is considered as an ideal matrix for static extractions without any binding matrix. Here, numerical simulations were based on the mass transfer kinetics of analytes that take place between the boundary layer of the SPME fiber coating and the sample matrix. The mass balance equation can be expressed as:

$N_{i}=-\nabla \cdot(D \nabla c)+\boldsymbol{u} \cdot \nabla c+R$

Where $\mathrm{c}$ is the concentration of the species $\left(\mathrm{mol} . \mathrm{m}^{-3}\right), D$ denotes the diffusion coefficient $\left(\mathrm{m}^{2} . \mathrm{s}^{-1}\right), R$ is the amount of any species produced or consumed in the system $\left(\mathrm{mol} \cdot \mathrm{m}^{-3} \cdot \mathrm{s}^{-1}\right)$, and $\boldsymbol{u}$ is the velocity vector $\left(\mathrm{m} \cdot \mathrm{s}^{-1}\right)$. The flux vector $N_{i}\left(\mathrm{~mol} \cdot \mathrm{m}^{-2} \cdot \mathrm{s}^{-1}\right)$ is associated with the net change in mass transfer at the boundary layer.

In static extraction conditions for agar gel, $N_{i}$ is only controlled by diffusion and the binding equilibrium. 
$N_{i}=-D \nabla c$

For solid-coating adsorption kinetics, the flux vector $N_{i}$ for static extraction in agarose gel can be expressed as:

$N_{i}\left(m o l . m^{-2} \cdot s^{-1}\right)=r_{\text {ads }}-r_{\text {des }}$

From eq. (7) and (17),

$$
\frac{\partial c s}{\partial t}\left(m o l . m^{-2} \cdot s^{-1}\right)=k_{\text {ads }} *[A] *\left(1-\frac{c s}{\gamma_{\mathrm{s}}}\right)-k_{\text {des }} * \frac{c s}{\gamma_{\mathrm{s}}}
$$

Eq. (18) defines the mass transfer kinetics in static mode for solid coatings. The constant $k_{a d s}$ was experimentally determined based on the assumption that $r_{\text {des }}$ can be considered negligible at the start of extraction, since the amount of extract on the fiber is infinitesimally small. In this case, eq. (18) can be expressed as:

$\frac{\partial c s}{\partial t}\left(m o l . m^{-2} \cdot s^{-1}\right)=k_{a d s} *[A]^{e q} *\left(1-\frac{c s}{\gamma_{s}}\right)$

Experimentally, $\frac{\partial c}{\partial t}$ can be obtained from the amount extracted on the fiber over time when the extraction rate is in the linear regime, which was experimentally observed to occur after 10 mins of extraction. Prior to this time point, the amount of extract on the fiber was below the instrumental LOQ. Therefore, $k_{a d s}$ from eq. (19) can be derived as follows:

$$
k_{\text {ads }}\left(\frac{\mathrm{m}}{\mathrm{s}}\right)=\frac{\frac{\partial \mathrm{c}}{\partial t}}{[\mathrm{~A}]^{t} *\left(1-\frac{\mathrm{cs}}{\gamma_{\mathrm{s}}}\right)}
$$

Once $k_{a d s}$ is attained via eq. (22), the value of $k_{d e s}$ can be obtained from eq. (7), followed by measurement of the adsorption equilibrium constant $\mathrm{K}$. In this work, the specific surface area per fiber was $4.54 \mathrm{e}^{-5}\left(\mathrm{~m}^{2}\right)$, while the saturated amount of DOX extracted per fiber was determined as $5.1 \mathrm{e}^{-09}$ (mol) (Fig S3). The calculated value of $\gamma_{\mathrm{s}}$ for DOX was $1.12 \mathrm{e}$ ${ }^{04}\left(\mathrm{~mol}_{\mathrm{m}} \mathrm{m}^{-2}\right)$. From eq. (7), the estimated value of the adsorption equilibrium constant for DOX was $468\left(\mathrm{~m}^{3} \cdot \mathrm{mol}^{-1}\right)(\mathrm{S} 4)$. Taking into account these parameters, the extraction kinetics were simulated and validated against experimental results.

Simulation was performed using a 3D model designed in COMSOL Multiphysics, using the experimental parameters listed above to demonstrate the extraction kinetics in agar gel. The calculated results were then compared with the experimental extraction time profile. The simulation showed that the equilibrium time for static extraction was approximately $80 \mathrm{~h}$, which is in good agreement with experimental results (see Figure 1). Detailed experimental conditions are given in the Supporting Information (section 5).

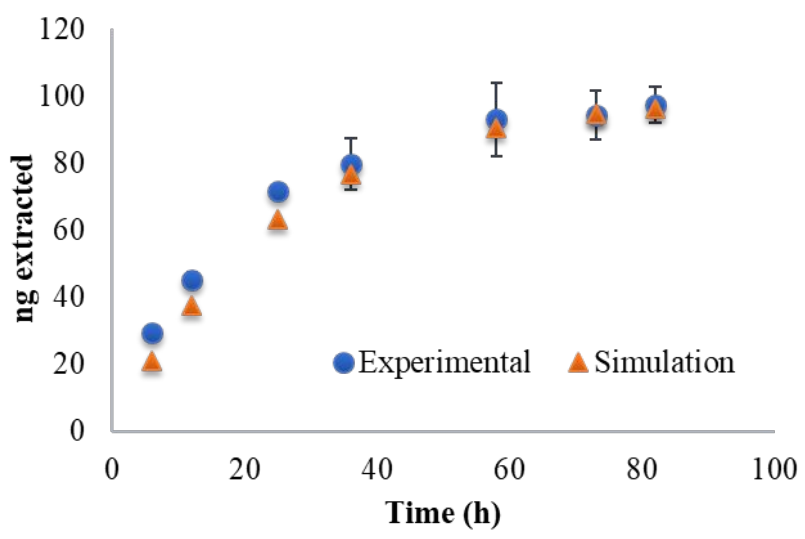

Figure 1 Comparison of in-silico and experimental extraction time profiles of DOX with an initial concentration of 100 $\mathrm{ng} \cdot \mathrm{mL}^{-1}$ in agarose gel.

In-Silico Study of Extraction Kinetics using Agitated Conditions (in PBS). Under agitation, the flux vector $N_{i}$ can be expressed as:

$N_{i}=-\nabla \cdot(D \nabla c)+\boldsymbol{u} \cdot \nabla c=r_{a d s}-r_{d e s}$

The mass transfer equation for solid coatings is the same as that stated in eq. (19), except that in this case, the flux $N_{i}$ changes rapidly due to the convection term $\boldsymbol{u}$, which enables equilibrium conditions to be established at a faster rate in comparison to the rate of equilibrium at static conditions. In the simulation, convection was considered as laminar flow velocity in order to avoid the high degree of nonlinearity associated with the mathematical function. In this case, we converted the vortex speed used $(1500 \mathrm{rpm})$ for experimental extraction into laminar flow velocity.

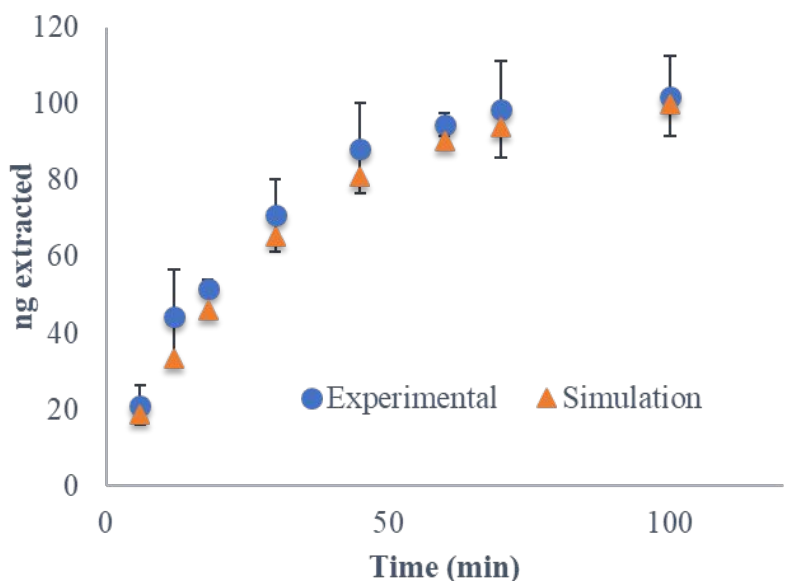

Figure 2 Comparison of in-silico and experimental extraction time profiles of DOX, with initial concentration of $100 \mathrm{ng} \cdot \mathrm{mL}$ ${ }^{1}$ in PBS.

Simulated extraction kinetics under agitated conditions forecasted the establishment of equilibrium at approximately $100 \mathrm{~min}$, which is in good agreement with experimental results (see Figure 2).

In-Silico Study of Extraction Kinetics under Agitated Conditions in the Presence of an HSA Binding Matrix. In the presence of a binding matrix, the flux vector $N_{i}$ is defined 
by eq. (15). The reaction term $R$ of eq. (15) can be derived from eq. (12).

$$
R=k_{f} *\left([A]^{\text {free }} *[M]^{\text {tot }}\right)+k_{r} *\left([A]^{\text {tot }}-[A]^{\text {free }}\right.
$$

Therefore, the flux for analyte in the presence of a binding matrix can be calculated as:

$$
\begin{aligned}
& N_{i}=-\nabla \cdot(D \nabla c)+\boldsymbol{u} \cdot \nabla c-k_{f} *\left([A]^{\text {free }} *[M]^{t o t}\right)+k_{r} *\left([A]^{\text {tot }}\right. \\
& \left.-[A]^{\text {free }}\right)
\end{aligned}
$$

Equations used to calculate extraction time profiles in the presence of a binding matrix are as follows:

$$
\begin{aligned}
& \frac{\partial c s}{\partial t}=-D \nabla c+\boldsymbol{u} . \nabla c-k_{f} *\left([A]^{\text {free }} *[M]^{t o t}\right)+k_{r} *\left([A]^{t o t}-\right. \\
& \left.[A]^{\text {free }}\right)
\end{aligned}
$$

In order to simulate an extraction time profile in the presence of the HSA binding matrix, the value of the binding association constant $\left(K_{A}\right)$ needs to be determined experimentally by using eq. (13). $K_{A}$ was calculated from the slope of Figure 3, yielding a value of $6203\left(1 . \mathrm{mol}^{-1}\right)$, which is in agreement with the value found in the literature. ${ }^{10}$ This calculated value was used to simulate an extraction time profile in the presence of a binding matrix, with good agreement found between simulated and experimental results. In this simulation, the value of the dissociation rate constant, $k_{r}$ with HSA for DOX was taken to be $0.5\left(\mathrm{~s}^{-1}\right)$ based on a previously reported study that targeted similar drugs. ${ }^{17}$ In-Silico Study in Tissue Given that extraction in tissue matrix occurs under static conditions, the kinetics of extraction are calculated using the following static conditions formulae. The mass balance equation for tissue can be expressed as:

$N_{i}=-\nabla \cdot(D \nabla c)+R$

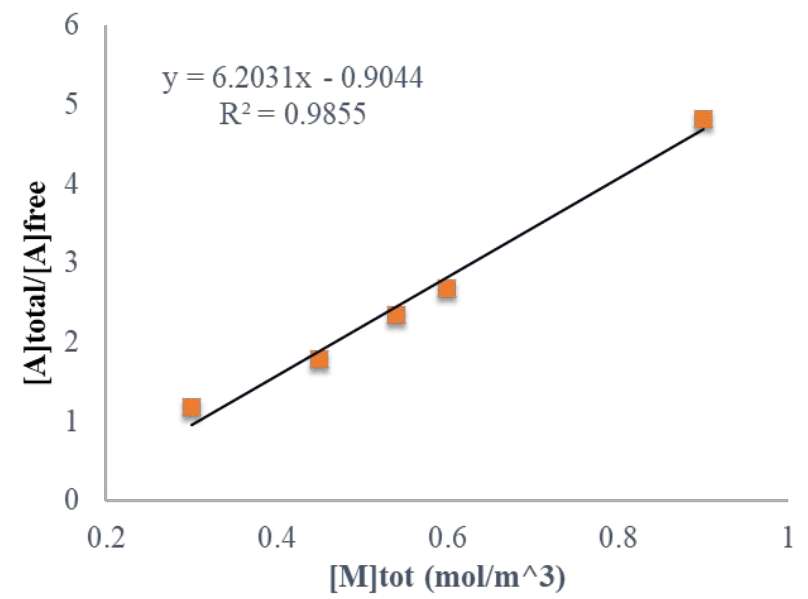

Figure 3 Ratio of bound and free analyte concentrations as a function of HSA concentration. Initial concentration of DOX was $100 \mathrm{ng} \cdot \mathrm{mL}^{-1}$.

Therefore, the flux $N_{i}$ can be expressed as:

$\frac{\partial c s}{\partial t}=-D \nabla c-k_{f} *\left([A]^{\text {free }} *[M]^{t o t}\right)+k_{r} *\left([A]^{t o t}-[A]^{\text {free }}\right)$ (26)

In the case of tissue matrix, an assumption based on eq. (14) is made to consider the apparent binding association constant
$K_{A p p}=K_{A \cdot[M]^{t o t}}$. The product of $K_{A \cdot[M]^{t o t}}$ is a unitless factor, and a constant for a given matrix. Here, $[M]^{\text {tot }}$ defines the total concentration of binding matrices. Since DOX has binding affinity to DNA, serum albumin, cell membrane, and other binding agents presents in the tissue matrix, calculating the true concentration of the binding matrix in tissue is therefore impractical due to the complex nature of the binding mechanism..$^{39,40}$ Therefore, we consider $K_{A p p}$ as an apparent binding constant, which can be predicted in a well-defined mathematical model by changing the total matrix concentration $[M]^{t o t}$. As the value of $K_{A}$ for DOX in the HSA matrix has been experimentally attained, $K_{A p p}$ can thus be estimated, yielding a value of $\mathbf{1 2 5 2}$ from a mathematical model fitted with the experimentally attained extraction time profile. This hypothesis was validated theoretically via simulations, where extraction kinetics were performed with different $K_{A}$ and $[M]^{\text {tot }}$ values for the same $K_{A p p}$. Simulated results showed similar extraction kinetics behavior which is not affected by the individual value of $K_{A}$ and $[M]^{\text {tot }}$ for a given $K_{A p p}$ value (see Figure 4). This observation provided by mathematical simulation simplified the complexities associated with experimental conditions, particularly when it becomes cumbersome to differentiate the binding association constant for each different binding matrix present in the sample.

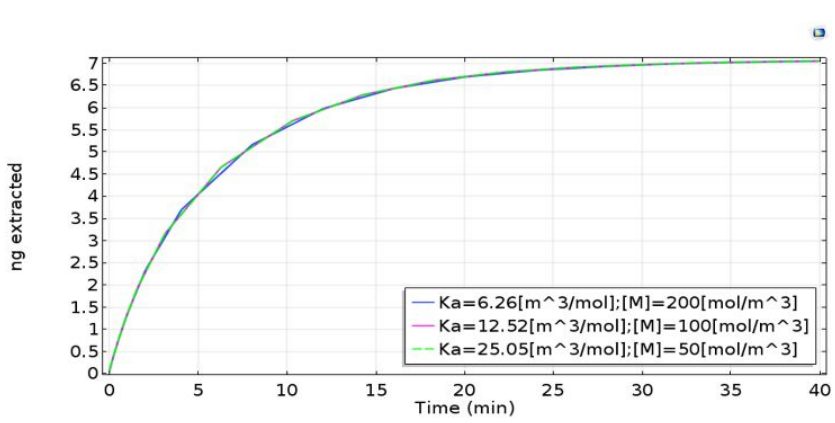

Figure 4 In-silico investigation of the apparent binding constant $K_{A p p}$. The extraction kinetic profiles behaved similarly despite using different matrix concentrations $[\mathrm{M}]$ and binding association constants $K_{A}$ for the same $K_{A p p}$. Initial concentration $[A]^{\text {tot }}$ was $25 \mu \mathrm{g} \cdot \mathrm{g}^{-1}$.

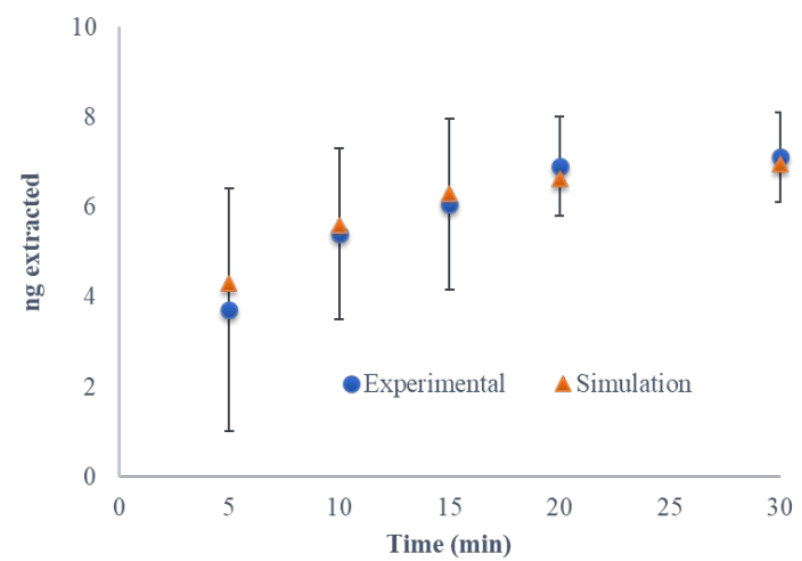

Figure 5 Comparison of in-silico and experimental extraction time profiles of doxorubicin $\left(25 \mu \mathrm{g} \cdot \mathrm{g}^{-1}\right)$ in homogenized bovine lung tissue. 
Experimental results from tissue showed that approximately 7 ng of DOX are extracted when an initial concentration of 25 $\mu \mathrm{g} \cdot \mathrm{g}^{-1}$ is spiked into tissue. As Figure 5 shows, extraction reached equilibrium after $25 \mathrm{~min}$ (equilibration time was herein defined as the time needed for $95 \%$ of the total equilibrium concentration to be extracted onto the coating), which is reasonable given the high binding affinity of DOX for lung tissue components. Simulated results were well-fitted with experimental results despite the fact that experimental errors are higher at lower time points due to the proximity of the experimentally extracted amount to experimental LOQ values. For all other studied initial concentrations in tissue matrix, the amount of extract at equilibrium fitted linearly to in-silico extracted amounts, while the analytical concentration of the sample was observed in the range studied. In-silico results were in agreement with the attained experimental results. ${ }^{35}$ Finally, the free concentration of DOX from lung tissue was estimated from eq. (14), yielding a value of $1.25 \mathrm{e}^{-05}\left(\mathrm{~mol}^{-3} \mathrm{~m}^{-3}\right)$ for $25 \mu \mathrm{g} \cdot \mathrm{g}$ ${ }^{1}$. These results indicate that DOX is heavily bound to the tissue matrix, at an estimated binding percentage of $99.97 \%$. The experimentally attained value was very close to the value generated by numerical simulations.

Negligible Depletion of Free Concentration and Spatial Resolution To determine the free concentration of analyte in the presence of binding matrix components, negligible depletion of the free concentration surrounding the SPME fiber is imperative. Figure 6 (a) describes how the free concentration DOX depletes over the time around the SPME fiber at static extraction in agar gel. The $\mathrm{X}$ axis represents the distance from the SPME fiber in millimeter while the $\mathrm{Y}$ axis is the concentration of DOX in the sample matrix in mol. $\mathrm{m}^{-3}$. At the beginning of extraction time profile, the depletion of concentration in the proximity of the fiber is higher (the green line graph at $1 \mathrm{~h}$ ). The total length of the $X$ axis is considered as the dimension of the sample matrix which is the diameter of the glass vial $(10 \mathrm{~mm})$ used in the experiment. From that figure, we can assume that the boundary layer thickness is approximately $8 \mathrm{~mm}$ after $1 \mathrm{~h}$ of extraction. However, close to the equilibrium (the blue line graph at $82 \mathrm{~h}$ ) there is no gradient of concentration around the fiber. On the other hand, extraction from tissue matrix apparently exhibited negligible depletion of the free concentration at equilibrium conditions, as seen in Figure 6 (b). Therefore, although the C-8 mixed mode SPME fiber enabled significant depletion of the free concentration of DOX at equilibrium, estimated at approximately 60 percent of the initial concentration, extraction from tissue matrix apparently exhibited negligible depletion of the free concentration at equilibrium conditions. Such a phenomenon enabled by the rapid release of DOX from the matrix-bound complex, indicating that the binding matrix and the SPME extraction phase compete for DOX. In fact, the binding matrix works as a buffer to maintain the free form of DOX surrounding the fiber while it is being extracted onto the extraction phase. It should be noted as well that the free concentration of DOX will likely not change in the matrix system unless the dissociation rate constants $k_{r}$ are slow. ${ }^{17}$ This also indicates that successive extractions from the same place within the sample matrix should not matter particularly when the percentage of the matrix bound complex is significantly high, as the matrix replenishes the free concentration rapidly. Figure 6 (c) evidences the spatial resolution of the SPME sampling by showing the depleted DOX concentration volume surrounding the fiber at equilibrium. This indicates that placing multiple SPME fibers in a small region of sample matrix should not interfere in the extraction kinetics. Therefore, experimental results should incur less bias relative to the matrix.

\section{CONCLUSIONS}

This article theoretically studies the extraction dynamics of molecules from bovine lung tissue using solid-phase microextraction, further presenting experimental validation of the COMSOL simulation models using, as a proof of concept, a well-known chemotherapeutic drug, doxorubicin (DOX) as a model compound. In this context, a novel technique was introduced for measurement of free drug concentration in bovine tissue homogenate by using biocompatible C- 8 mixed mode SPME fiber. Also, theoretical studies were discussed and defined to interpret several crucial concepts on mass transfer kinetics that take place in tissue matrix. To validate the established mathematical models, a novel SPME technique to determine free and bound concentrations from tissue by either in vivo or ex vivo sampling was theoretically developed and experimentally established, using DOX. The attained experimental results were explained and cross-validated by mathematical COMSOL simulations, which described in detail the mass transfer kinetics of DOX in lung tissue matrix. Moreover, derived from this primary work, several relevant concepts concerning tissue sampling, such as apparent binding constant, spatial resolution, and local depletion, were developed and discussed. Of note, the attained results help to shed light on the principle of SPME tissue extraction, including in vivo extraction scenarios. As part of this investigation, the unbound free concentration of analyte is shown to remain constant when a thin coating SPME fiber is used, as the amount of analyte that is transferred to the coating is replaced from the reservoir of analyte bound to the tissue matrix. 

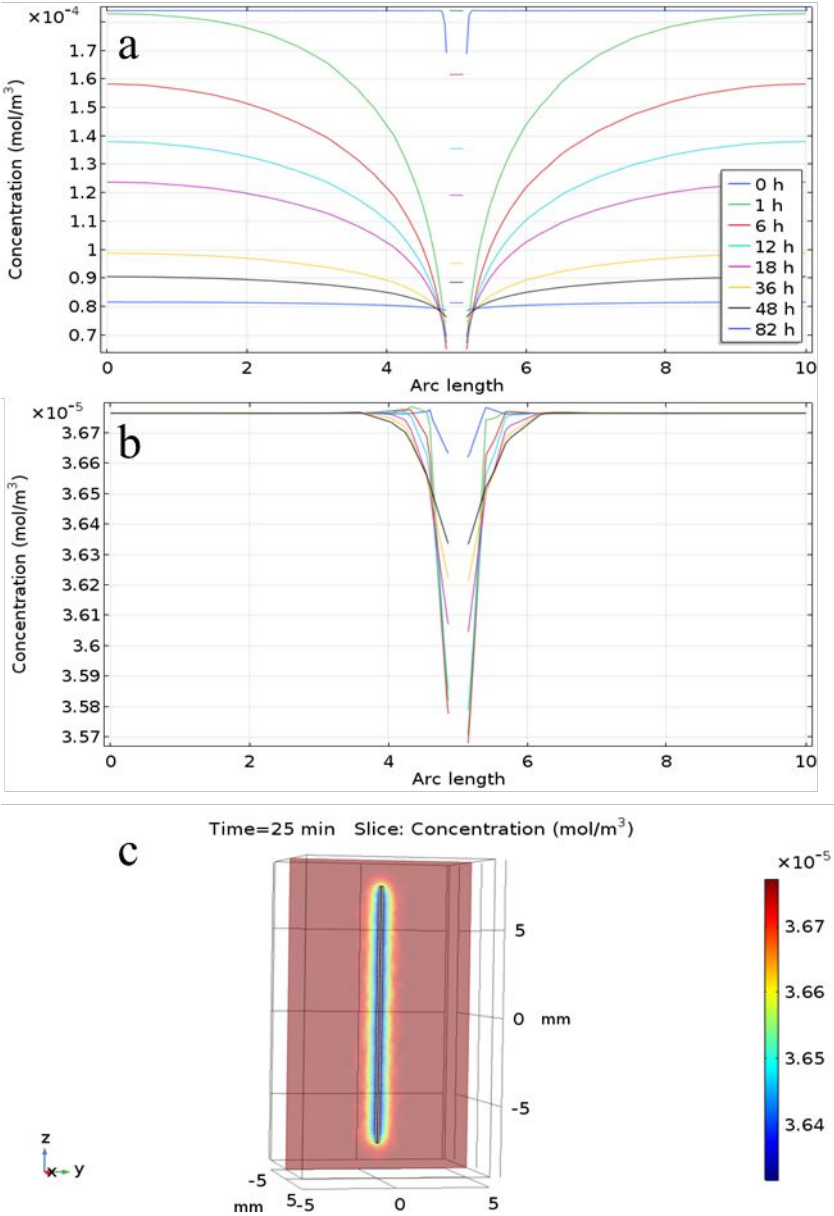

Figure 6 (a) COMSOL simulation shows the change of DOX concentration in agarose gel across a line drawn through the center of the SPME fiber at different extraction times. The $\mathrm{X}$ axis shows the distance from the fiber, and the $\mathrm{Y}$ axis represents concentration. The initial concentration was $0.1 \mu \mathrm{g} \cdot \mathrm{mL}^{-1}$ (b) The change in the free concentration of DOX in tissue at equilibrium, indicating that the gradient in concentration is negligible. The initial concentration was $25 \mu \mathrm{g} \cdot \mathrm{g}^{-1}$. (c) The spatial resolution of the concentration gradient of DOX across a $2 \mathrm{D}$ line cut through the SPME fiber.

In addition, the presented work represents the first attempt to measure free drug concentrations in tissue matrix with the assistance of mathematical models. The key attributes of the herein presented numerical models include estimation of the apparent binding constant $\mathrm{K}_{\mathrm{App}}$ of DOX in tissue matrix, demonstration of negligible depletion, and spatial resolution for multiple ex vivo or in vivo samplings. Here, $\mathrm{K}_{\mathrm{App}}$ is defined as a unitless constant that describes the binding properties of an analyte in a specific sample matrix; in this work, the $\mathrm{K}_{\mathrm{App}}$ for DOX in bovine lung tissue was estimated as 1252 . The value of $\mathrm{K}_{\text {App }}$ is difficult to estimate experimentally since the total concentration of binding species $[\mathrm{M}]$ is unknown in a given tissue matrix. This limitation is herein addressed by taking advantage of the mathematical models established in this work. Although this study was performed using homogenized tissue, future research efforts may include estimation of in vivo free concentrations in the extracellular space of tissue matrix. The developed simulation approach thus substantiates that SPME can be used as a valuable tool for accurate measurements of free drug concentrations in tissue matrix. Of note, we could have selected BSA as a binding matrix to estimate the binding association constant $\mathrm{K}_{\mathrm{A}}$ instead of HSA. However, given that the ultimate goal of this ongoing research concerns future implementation of this technique for in vivo determinations in human lung tissue, HSA was herein selected as binding matrix. Finally, the output of this article is not only limited to DOX or lung tissue, but also paves the way for quantitation of free and conjugated forms of other drugs in any complex biological matrix. In fact, this study will allow for exploration of completely new horizons in biomedical sciences, such as in vivo monitoring of drug pharmacodynamics in tissue, without the need of correlative investigations using surrounding biofluids or biopsies.

\section{ASSOCIATED CONTENT}

\section{Supporting Information}

The supporting information for this document includes the method used for standard sample preparation in PBS, measurement of the active surface area of the SPME mixed mode fiber, measurement of the maximum site concentration $\gamma_{\mathrm{s}}$ s, experimental measurement of the adsorption equilibrium constant $K$, and tables of parameters used for in silico studies.

\section{AUTHOR INFORMATION}

Corresponding Author

* Phone: +1 (519) 888 4641; Fax: +1 (519) 7460435.

E-mail: janusz@uwaterloo.ca

Notes

The authors declare no competing financial interest.

\section{Current affiliation}

$¥$ Instituto de Investigación e Ingeniería Ambiental (3iA), Universidad Nacional de San Martín (UNSAM), San Martín, Buenos Aires, Argentina.

† Department of Pharmaceutical Chemistry, Medical University of Gdańsk, Gdańsk, Poland.

\section{ACKNOWLEDGMENTS}

The authors are grateful to Supelco (Sigma Millipore), Thermo Scientific (USA) and the Natural Sciences and Engineering Research Council (NSERC) of Canada for the financial support provided through the Industrial Research Chair program.

\section{REFERENCES}

(1) Zou, L. Importance of Drug Pharmacokinetics at the Site of Action. 2017, No. November 2016, 133-142.

(2) Schmidt, S.; Banks, R.; Kumar, V. Clinical Microdialysis in Skin and Soft Tissues: An Update. 2008, 351-364.

(3) Revue, L.; Industries, P.; Metabolic, T.; Commission, T. E.; European, T. The Effect of Plasma Protein Binding on in Vivo Efficacy: Misconceptions in Drug Discovery. 2010, 9 (DECEmbER), 929-939.

(4) Reck dos Santos, P.; Sakamoto, J.; Chen, M.; Linacre, V.; Arce, C.; Liu, M.; Waddell, T. K.; Keshavjee, S.; Cypel, M. Modified In Vivo Lung Perfusion for Local Chemotherapy: A Preclinical Study With Doxorubicin. Ann. Thorac. Surg. 2016, 101 (6), 2132-2140.

(5) Collins, J. M.; Klecker, R. W. Evaluation of Highly Bound Drugs : Interspecies, Intersubject, and Related Comparisons. 2002, 902, 971-975.

(6) Hoseyni, H.; Xu, Y.; Zhou, H. Therapeutic Drug Monitoring of Biologics for Inflammatory Bowel Disease: An Answer to 
(15) Phase, S.; Fused, U. Solid Phase Microextraction with Thermal Desorption Using Fused Silica Optical Fibers. 1990, 2145-2148.

(16) Pawliszyn, J. Handbook of Solid Phase Microextraction, Chemical I.; Beijing, 2009.

(17) Alam, M. N.; Ricardez-Sandoval, L.; Pawliszyn, J. Numerical Modeling of Solid-Phase Microextraction: Binding Matrix Effect on Equilibrium Time. Anal. Chem. 2015, 87 (19), 9846-9854.

(18) Musteata, F. M. Monitoring Free Drug Concentrations : Challenges. 2011, 1753-1768.

(19) Lord, H. L.; Zhang, X.; Musteata, F. M.; Vuckovic, D.; Pawliszyn, J. In Vivo Solid-Phase Microextraction for Monitoring Intravenous Concentrations of Drugs and Metabolites. Nat. Protoc. 2011, 6 (6), 896-924.

(20) Musteata, F. M.; de Lannoy, I.; Gien, B.; Pawliszyn, J. Blood Sampling without Blood Draws for in Vivo Pharmacokinetic Studies in Rats. J. Pharm. Biomed. Anal. 2008, 47 (4-5), $907-$ 912.

(21) Lord, H. L.; Grant, R. P.; Walles, M.; Incledon, B.; Fahie, B.; Pawliszyn, J. B. Development and Evaluation of a Solid-Phase Microextraction Probe for in Vivo Pharmacokinetic Studies. Anal. Chem. 2003, 75, 5103-5115.

(22) Bojko, B.; Gorynski, K.; Gomez-rios, G. A.; Matthias, J.; Machuca, T.; Nikolaus, V.; Cudjoe, E.; Hsin, M.; Cypel, M.; Selzner, M.; et al. Analytica Chimica Acta Solid Phase Microextraction Fills the Gap in Tissue Sampling Protocols. Anal. Chim. Acta 2013, 803, 75-81.

(23) Cudjoe, E.; Bojko, B.; de Lannoy, I.; Saldivia, V.; Pawliszyn, J. Solid-Phase Microextraction: A Complementary in Vivo Sampling Method to Microdialysis. Angew. Chem. Int. Ed. Engl. 2013, 52 (46), 12124-12126.

(24) Ouyang, G.; Vuckovic, D.; Pawliszyn, J. Nondestructive Sampling of Living Systems Using in Vivo Solid-Phase Microextraction. Chem. Rev. 2011, 111 (4), 2784-2814.

(25) Vuckovic, D.; De Lannoy, I.; Gien, B.; Shirey, R. E.; Sidisky, L. M.; Dutta, S.; Pawliszyn, J. In Vivo Solid-Phase Microextraction: Capturing the Elusive Portion of Metabolome. Angew. Chemie Int. Ed. 2011, 50 (23), 5344-5348.
(26) Musteata, F. M.; Pawliszyn, J. Study of Ligand-Receptor Binding Using SPME: Investigation of Receptor, Free, and Total Ligand Concentrations. J. Proteome Res. 2005, 4 (3), 789-800.

Alam, M. N.; Ricardez-Sandoval, L.; Pawliszyn, J. Calibrant Free Sampling and Enrichment with Solid-Phase Microextraction: Computational Simulation and Experimental Verification. Ind. Eng. Chem. Res. 2017, 56 (13), 3679-3686.

Alam, M. N.; Pawliszyn, J. Effect of Binding Components in Complex Sample Matrices on Recovery in Direct Immersion Solid-Phase Microextraction: Friends or Foe? Anal. Chem. 2018 90 (4), 2430-2433.

(29) Górecki, T.; Yu, X.; Pawliszyn, J. Theory of Analyte Extraction by Selected Porous Polymer SPME Fibres $\dagger$. Analyst 1999, 124 (5), 643-649.

(30) Zhou, S. N.; Zhang, X.; Ouyang, G.; Es-haghi, A.; Pawliszyn, J. On-Fiber Standardization Technique for Solid-Coated SolidPhase Microextraction. Anal. Chem. 2007, 79 (3), 1221-1230.

(31) Tacar, O.; Sriamornsak, P.; Dass, C. R. Doxorubicin : An Update on Anticancer Molecular Action ,. 2013, 157-170.

(32) Carvalho, C.; Santos, R. X.; Cardoso, S.; Correia, S.; Oliveira, P. J.; Santos, M. S.; Moreira, P. I. Doxorubicin : The Good, the Bad and the Ugly Effect Doxorubicin: The Good, the Bad and the Ugly Effect. 2009, No. October.

(33) Track, F. An Ancestral Haplotype Defines Susceptibility to Doxorubicin Nephropathy in the Laboratory Mouse. 2006, 17961800

(34) Alam, M. N.; Nazdrajić, E.; Singh, V.; Tascon, M.; Pawliszyn, J. Effect of Transport Parameters and Device Geometry on Extraction Kinetics and Efficiency in Direct Immersion SolidPhase Microextraction. Anal. Chem. 2018, 90 (19), 1154811555.

(35) Roszkowska, A.; Tascon, M.; Bojko, B.; Goryński, K.; dos Santos, P. R.; Cypel, M.; Pawliszyn, J. Equilibrium Ex Vivo Calibration of Homogenized Tissue for in Vivo SPME Quantitation of Doxorubicin in Lung Tissue. Talanta 2018, 183 (November 2017), 304-310.

(36) Decout, A.; Dubernet, C.; Henry-Toulme, N. Mechanism of Doxorubicin Release from Polymeric Particles: An Interfacial Transfer Limited Process Affected by Serum Proteins. J. Colloid Interface Sci. 1996, 181 (1), 99-107.

(37) Wolak, D. J.; Thorne, R. G. Diffusion of Macromolecules in the Brain: Implications for Drug Delivery. Mol. Pharm. 2013, 10 (5), 1492-1504.

(38) Saghyan, A.; Lewis, D. P.; Hrabe, J.; Hrabetova, S. Extracellular Diffusion in Laminar Brain Structures Exemplified by Hippocampus. J. Neurosci. Methods 2012, 205 (1), 110-118.

(39) Wop, F. A. De; Demep, R. A.; Bet, D.; Katsz, V.; Kruijff, B. De. Characterization of the Interaction of Doxorubicin with ( Poly ) Phosphoinositides in Model Systems Evidence for Specific Interaction with Phosphatidylinositol-Monophosphate And. 1991, 288 (1), 237-240.

(40) Awasthia, S.; Frenkel, P.; Bellib, A. The Relationship Drug Resistance of Doxorubicin Binding to Membrane Lipids With. 1992, 63, 109-116. 
Table of Contents (TOC) Graphic

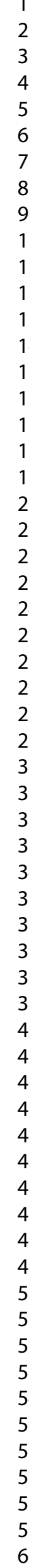

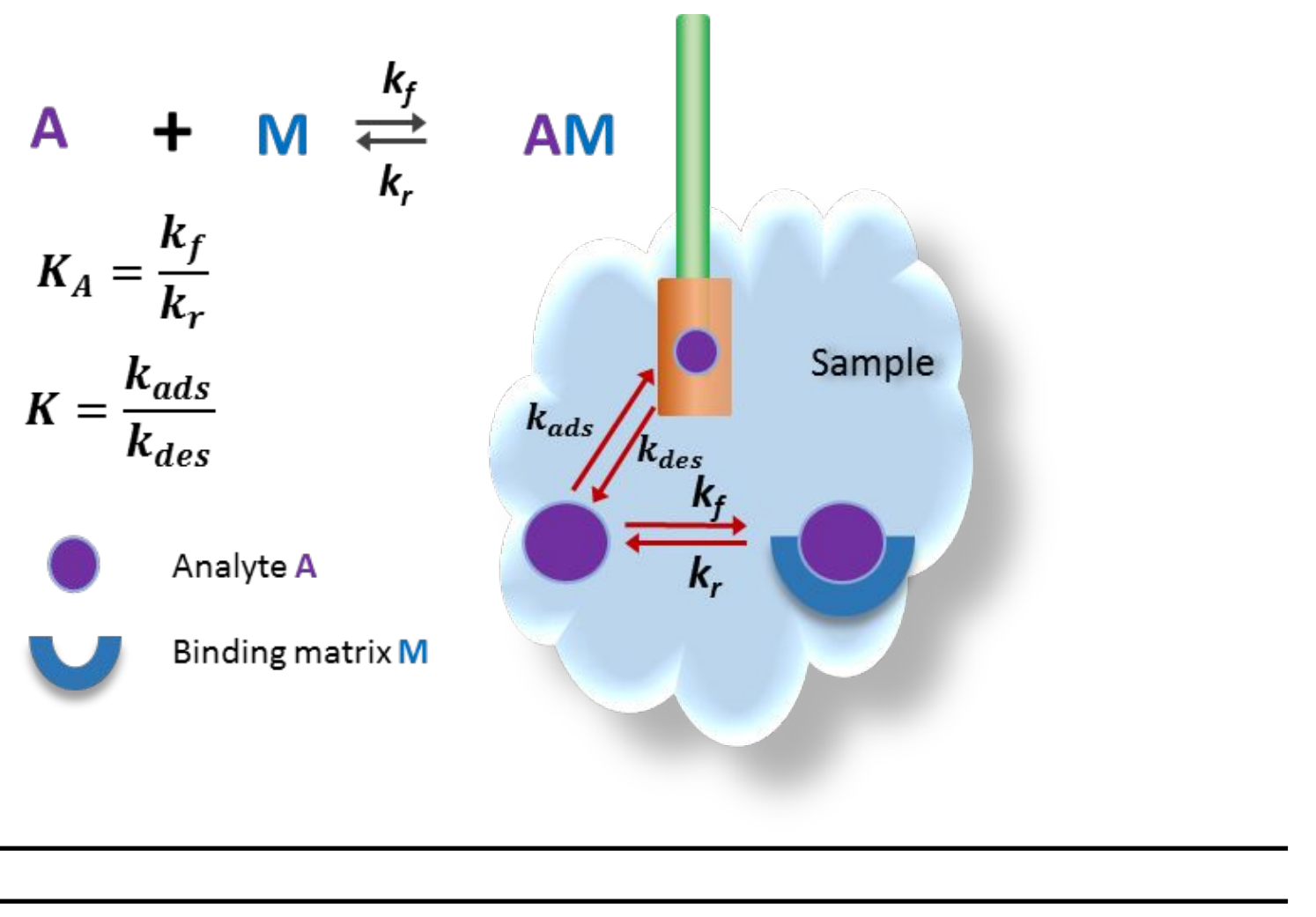

